\title{
Optimization of Cooperative Models in Cognitive Networks Using Genetic Algorithm
}

\author{
Nanduri V S S Ramya Jyothi, B. Leela Kumari
}

\begin{abstract}
The main theme of the $5 G$ radio otherwise called as Cognitive Radio is to utilize the spectrum to a licensed spectrum to the secondary users make this happen and to provide utmost efficiency. The allocation of spectrum holes of unutilized of this paper is to provide the primary and cognitive users optimal delay and throughput. A practical WC-policy-based algorithm is designed in sequence to closely approach the optimal value of the $S U$ delay where the secondary users are maximized in terms of traffic. With the optimized $S U$ delay the throughput of the primary user is also constrained. The trade-off between the parameters delay and throughput is attained by optimization problems using three different policies: WC policy, Non-WC policy and sub optimal policy. The trade-off is further improved by using the Genetic algorithm which is an evolutionary computational iterative process works on the principle of Darwin theory. This implementation had promoted better trade-off than the existing policies.
\end{abstract}

Keywords-Cognitive Radio, Delay, Genetic Algorithm, Optimization, Throughput, Trade-off

\section{INTRODUCTION}

The concept of cognitive radio came into existence by the impassion of ultimate usage of the spectrum available in spite of its scarcity. The $5 \mathrm{G}$ radio technology searches for the white spaces available for the efficient utilization of the resource. The radio comprises of primary users (PU) (licensed) who might not use the channel for all the time and the secondary users (SU) (unlicensed) who avails the spectrum holes after the licensed users. The Cognitive network consists of 3 stages: Observe, Decide and Act. The Observe cycle is where the spectrum holes are explored with accuracy, appropriate range and timing sense. The deciding stage is to decide about the distribution and usage of the spectrum resources by the decision-making processor also called as "Heart of Cognitive Radio". The act step is all about making the necessary observation and decision needed to be taken. The following figure 1 illustrates the concept of cognitive radio. Figure 2 shows the cycle of $5 \mathrm{G}$ radio. The performance of the detection algorithm is calculated on two parameters: The probability of false alarm $\mathrm{P}_{\mathrm{F}}$ and the probability of the detection $\mathrm{P}_{\mathrm{D}}$. However, the probability depends on the accuracy of signal and noise power estimation.

Revised Manuscript Received on 10, September 2019.

Nanduri V S S Ramya Jyothi, Electronics and Communication Engineering, JNT University, Telangana, India (E-Mail: nvss.sairamya@gmail.com)

B. Leela Kumari, Assistant Professor, Electronics And Communication Engineering, JNT University, Telangana, India (E-Mail: leela8821@yahoo.com)

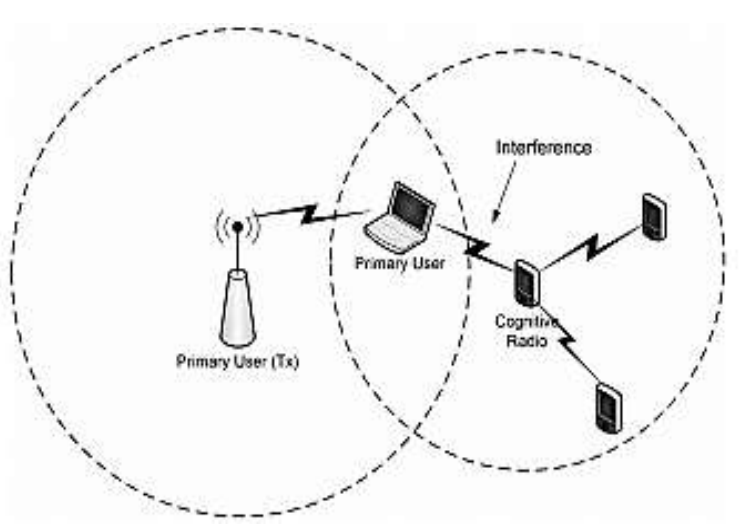

Figure 1: Concept of Cognitive radio

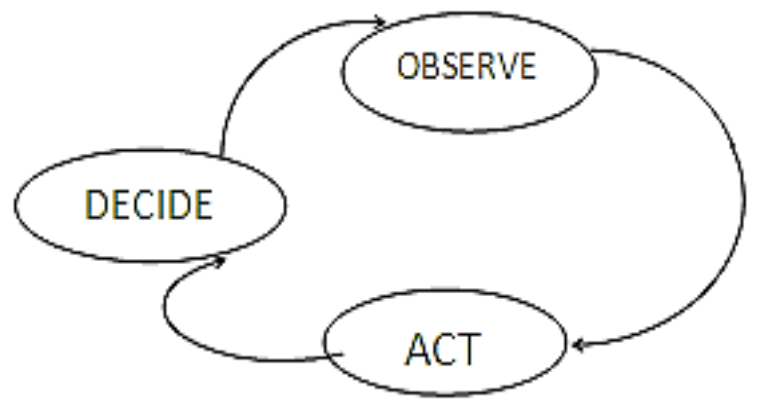

Figure 2: Cognitive Cycle

\section{OPTIMIZATION IN COGNITIVE RADIO}

According to Doyle "An optimization process involves selecting the best choice from the list of available choices in order to reach some kind of goal or at least get as near as possible to the goal". The optimization of a cognitive radio can be performed on allocation of the spectrum, power control and the Quality of service (QoS) i.e., the delay throughput tradeoff. The Optimization of delay and throughput is considered in this paper and it is optimized through Genetic algorithm (GA) and compared to the existing cooperative models that is work conservative policy and the non - work conservative policy.

\section{SYSTEM ORGANIZATION}

In this paper we have considered the cognitive radio network as sketched as below figure 3. The network incorporates of two users i.e., Primary users (PU) and secondary users (SU) and a standard destination. The PU is furnished with a queue, $Q_{p}$ for the primary user packets and likewise $\mathrm{SU}$ is furnished with a queue, $\mathrm{Q}_{\mathrm{s}}$ meant for the secondary user packets and the $\mathrm{Q}_{\mathrm{sp}}$ which is intended for the packets

Published By:

Blue Eyes Intelligence Engineering \& Sciences Publication 


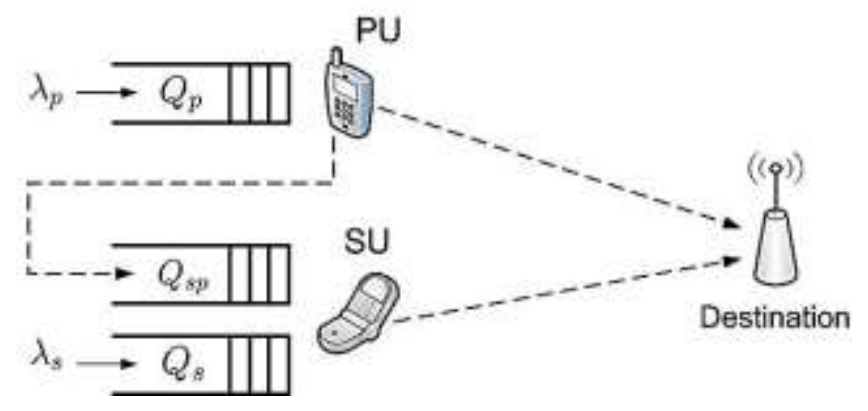

Figure 3: System organization

over headed from the PU packets. The packet arrival rates are defined as $\lambda_{\mathrm{p}}$ and $\lambda_{\mathrm{s}}$ using Bernoulli random process where $0 \leq \lambda_{\mathrm{p}} \leq 1$ and $0 \leq \lambda_{\mathrm{p}} \leq 1$.

The evolution of the length of the $\mathrm{j}^{\mathrm{t}}$ queue is characterized as

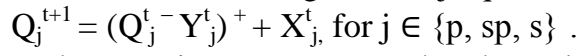

When a primary user uses the channel to propagate the packets through $\mathrm{Q}_{\mathrm{p}}$ towards the destination, the packets are received by the destination a positive acknowledgment (ACK) is send to PU indicating that the packets are received successfully. In this scenario we assume that the system is interference free channel by using the high detection probability detectors at the MAC layer. The probability of successful packet reception i.e., the probability of no linkage outage, between the primary user (PU) and the destination, the secondary user (SU) and the destination, and the PU and the $\mathrm{SU}$ are indexed by $\mathrm{h}_{\mathrm{pd}}, \mathrm{h}_{\mathrm{sd}}$ and $\mathrm{h}_{\mathrm{ps}}$, respectively. In this layer the PU can be detected for its Idle or the busy states using the detecting sensors at the secondary users.

\subsection{When the $P U$ is reserved}

Basically, when the PU transmittes the packets towards the destination, there exists three probable cases:

(i) If the destination receives the packets and sends back an acknowledgement to the PU that the packets are decoded perfectly by the destination nevertheless of whether the SU decoded the packets or not.

(ii) If the packets are decoded by the SU as well as the destination, then the packets are stored in $\mathrm{Q}_{\mathrm{SP}}$ with probability denoted by ' $a$ '. If accepted by the SU then it acknowledges the source and the packets are released from the $\mathrm{Q}_{\mathrm{p}}$.

(iii) If neither the SU nor the destination, then the packets are remained in the $\mathrm{Q}_{\mathrm{p}}$ for retransmission.

\subsection{When $P U$ is not busy}

If the channel is occupied by the $\mathrm{SU}$ through $\mathrm{Q}_{\mathrm{s}}$ with the probability ' $b$ ' or from the queue $Q_{s p}$ with probability ' $1-b$ '. Otherwise the packets are dropped, and the PU is idle. The condition that the PU is busy, and the slot is underutilised is said to be the WC cooperative policy.

\section{GA AND ITS ADVANTAGES}

The genetic algorithm is an efficient optimizing algorithm and also intelligent random search technique utilising the natural selection and the genetics. the algorithm involves five steps in optimizing the process. They are initial population, selection, mating, crossover and mutation. Initially we have generated an initial population of individuals and we evaluate the fitness of all the values. Then the selection starts to select the fittest individuals for further steps to be followed. The mating process takes place and again the fittest of all the new population and they are further generated.

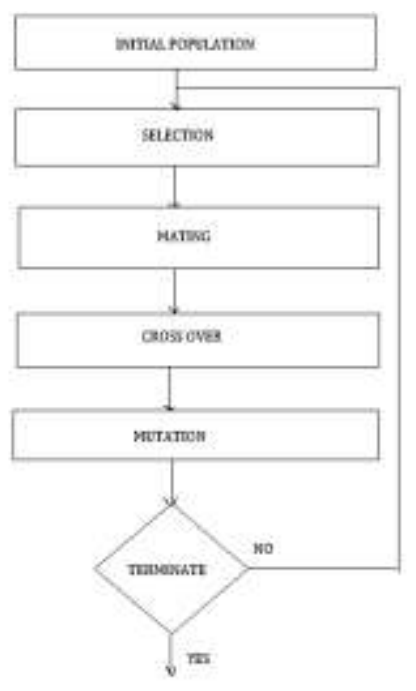

Figure 4: Flow chart showing steps to implement the genetic algorithm

Coming to the advantages of genetic algorithm (GA) is that the selection process enables to search the solution space faster.

\section{IMPLEMENTATION OF IDEA \& RESULTS}

The service rates of the PU and SU are formulated as below from the service rate of $Q_{p}$ is $\mu_{p}, \mu_{p}=h_{p d}+\left(1-h_{p d}\right) h_{p s}$ a and similarly the service rate of $Q_{\mathrm{s}}$ is $\mu_{\mathrm{s}}$,

$$
\mu_{\mathrm{s}}=\mathrm{b} \mathrm{h}_{\mathrm{sd}}\left(1-\lambda_{\mathrm{p}} / \mu_{\mathrm{p}}\right)
$$

whereas the service rate of $\mathrm{Q}_{\mathrm{sp}}$ is formulated as

$$
\mu_{\mathrm{sp}}=(1-\mathrm{b}) \mathrm{h}_{\mathrm{sd}}\left(1-\lambda_{\mathrm{p}} / \mu_{\mathrm{p}}\right) \text {. }
$$

The average queue lengths of the $\mathrm{Q}_{\mathrm{s}}, \mathrm{Q}_{\mathrm{p}}$ and $\mathrm{Q}_{\mathrm{sp}}$ are indicated by $\mathrm{Np}$, Nsp and Ns individually. These queue lengths are derived from the application of Pollaczek-Khinchine formula [8] with Bernoulli arrival rate which is represented by $\lambda p$ using discrete time $M / M / 1$ queue in the matlab using communication tools.

The Inequalities of the stability of the queue is formulated such that the network would be operated accordingly. That is

$$
\lambda_{\mathrm{p}}<\mu_{\mathrm{p}}, \lambda_{\mathrm{s}}<\mu_{\mathrm{s}}, \lambda_{\mathrm{sp}}<\mu_{\mathrm{sp}}
$$

Likewise, the average delays of the primary and cognitive user are formulated using little's law such that

$$
\mathrm{Dp}=(\mathrm{Np}+\mathrm{Nsp}) / \lambda \mathrm{p}, \mathrm{Ds}=\mathrm{Ns} / \lambda \mathrm{s}
$$

For defining the secondary user throughput, the optimization problem is considered as follows. Here we optimized the problem of maximizing the SU throughput with the PU delay constraint. Here is the formula that the problem is framed: 
Q1: $\max \quad \operatorname{bh}_{\mathrm{sd}}\left(1-\left(\lambda \mathrm{p} / \mu_{\mathrm{p}}\right)\right)$

$a, b$

s.t. $0 \leq \mathrm{a} \leq 1$,

$0 \leq \mathrm{b} \leq 1$,

$\mu \mathrm{p}=\mathrm{h} p d+(1-\mathrm{h} \mathrm{pd}) \mathrm{h}_{\mathrm{ps}} \mathrm{a}$,

$$
\left(\mathrm{N}_{\mathrm{p}}+\mathrm{N}_{\mathrm{sp}}\right) / \lambda \mathrm{p} \leq \psi[8]
$$

The paper is mainly focused on the concept to improve the QoS even with cognitive user while using the applications like Voice over internet protocol, live streaming and some other media applications like screen sharing etc. Parallelly providing the licensed user with the efficient communication channel.

The suboptimal policy and the WC policy are principled with the conditions such as arrival rates and the probabilities of the channel as follows: $\mathrm{h}$ pd $=0.3$, h ps $=0.4$, hsd $=0.8$. Now coming to the Primary delay constraint, the Threshold limit plays a important role where $\psi=10$. The problem Q1 is a non-convex function which is converted to quasi convex function to solve in an iterative process with $\psi=10$ and $\lambda_{\mathrm{p}}=$ 0.2 and $\lambda_{\mathrm{s}}=0.4$.

Summary of results:

The following are the graphical representations of the average delay packet versus arrival rate at SU packets, the stable throughput region graphs and the graph that illustrates the average packet delay of the lincensed and unlicensed users.

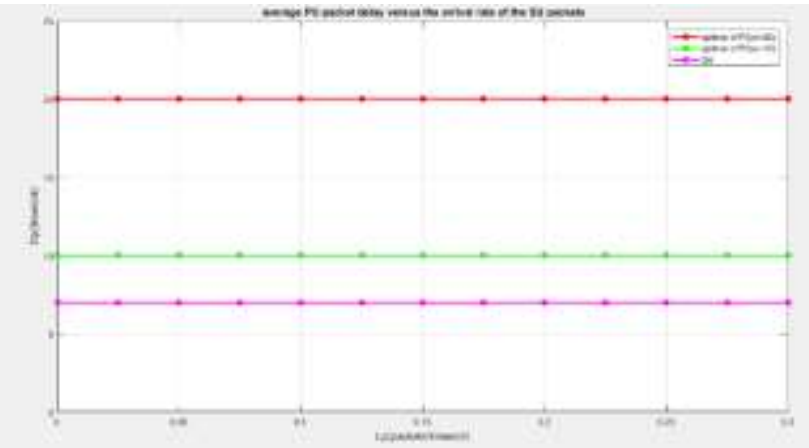

Figure 5: Graph illustrating the average PU packet delay Vs average $\mathrm{SU}$ packet dealys comparing with the implementation of GA.

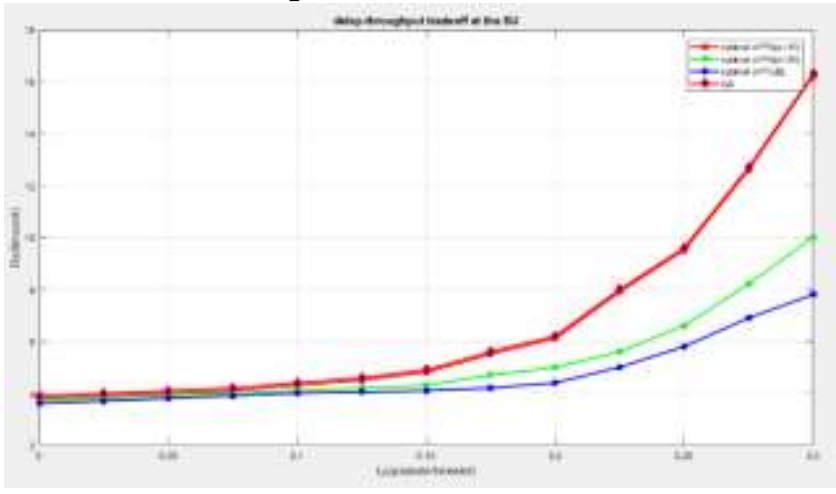

Figure 6: Graph illustrating the Delay and throughput trade off at the $\mathrm{SU}$ considering lincensed users Delay constraint with a $\psi=10$.

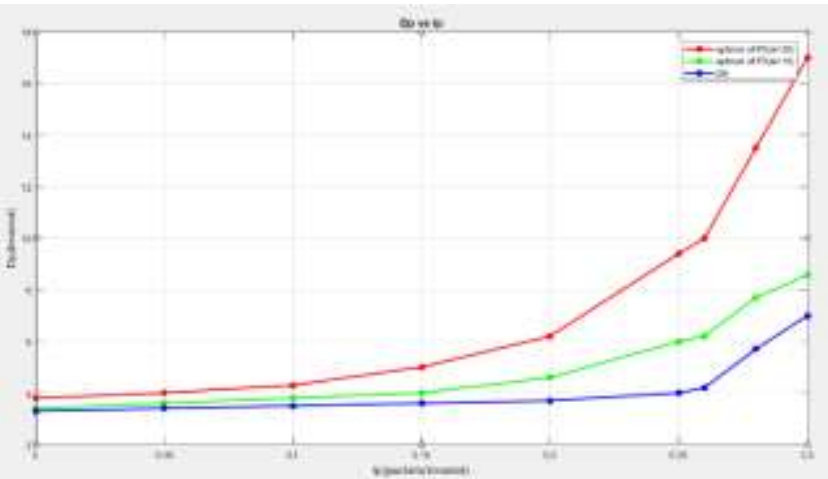

Figure 7: Graph that illustrating the Delay of PU Vs arrival rate on $\mathrm{PU}$ with predefined conditions and threshold.

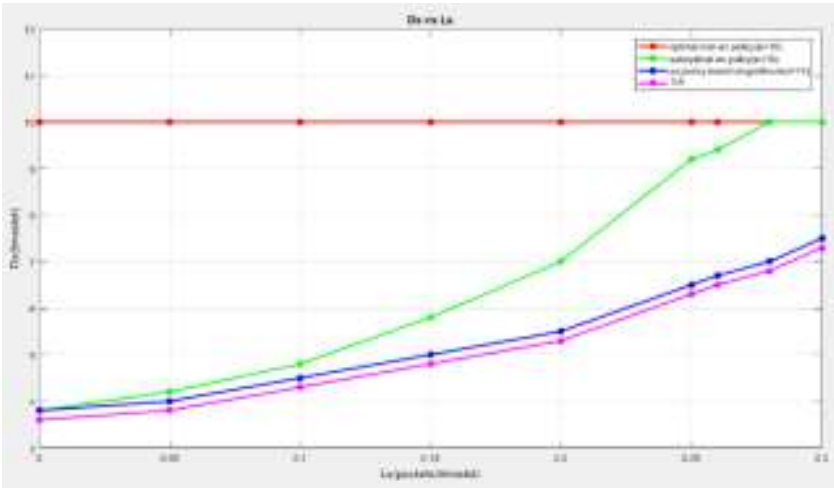

Figure 8: Graph that illustrates the arrival rate and the delay on SU with predefined conditions and threshold.

\section{CONCLUSION AND FUTURE SCOPE}

In the present approach, we mainly concentrated on Delay Throughput trade-off is exploited using the optimization problem using iterative manner and then optimizing using fitness values This approach can further be extended by different optimizing algorithms such as enhanced stimulated annealing algorithm, particle swarm optimization and optimization can be improved further more.

\section{REFERENCES}

1. Adel M. Elmahdy, Amr El-Keyi, Tamer ElBatt, Senior Member, IEEE, and Karim G. Seddik, Senior Member, "Optimizing Cooperative Cognitive Radio Networks Performance with Primary QoS Provisioning", IEEE TRANSACTIONS ON COMMUNICATIONS, VOL. 65, NO. 4, APRIL 2017.

2. S. Haykin, "Cognitive radio: Brain-empowered wireless communications,” IEEE J. Sel. Areas Commun., vol. 23 , no. 2, pp. 201-220, Feb. 2005.

3. M. Ashour, A. A. El-Sherif, T. ElBatt, and A. Mohamed, "Cognitive radio networks with probabilistic relaying: Stable throughput and delay tradeoffs," IEEE Trans. Commun., vol. 63, no. 11, pp. 4002-4014, Nov. 2015.

4. O. Simeone, Y. Bar-Ness, and U. Spagnolini, "Stable throughput of cognitive radios with and without relaying capability," IEEE Trans. Commun., vol. 55, no. 12, pp. 2351-2360, Dec. 2007. 
5. Newman, T R, R Rajbanshi, A M Wyglinski, J B Evans, and $\mathrm{G}$ J Minden. "Population Adaptation for Genetic Algorithm based Cognitive Radios." 2nd International Conference on Cognitive Radio Oriented Wireless Networks and Communications. Orlando, FL: IEEE, 2007. 279-284.

6. Miguel Luís, Rodolfo Oliveira, Rui Dinis, Luis Bernardo, "RF-spectrum opportunities for cognitive radio networks operating over GSM channels" IEEE Transactions on cognitive communications and networking, vol. 3 , no. 4 , December 2017.

7. A. M. Elmahdy, A. El-Keyi, T. ElBatt, and K. G. Seddik, "On the stable throughput of cooperative cognitive radio networks with finite relaying buffer," in Proc. IEEE Int. Annu. Int. Symp. Pers. Indoor Mobile Radio Commun. (PIMRC), Sep. 2014, pp. 942-946.

8. L. Kleinrock, Queueing Systems: Theory, vol. 1. New York, NY, USA: Wiley, 1975.

9. A. S. Ibrahim, A. K. Sadek, W. Su, and K. R. Liu, "Cooperative communications with relay-selection: When to cooperate and whom to cooperate with?" IEEE Trans. Wireless Commun., vol. 7, no. 7, pp. 2814-2827, Jul. 2008.

\section{AUTHORS PROFILE}

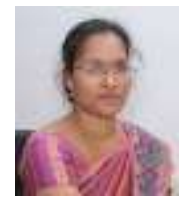

Leela Kumari B received B.Tech from JNT University, M. Tech from Andhra University, Ph.D. from JNT University. She has 16 years of teaching experience and is Assistant Professor in JNT University. She has published more than 60 technical papers in National/International Journals/Conference proceedings. Her research interests include Signal processing, State Estimation, tracking and particle filters.

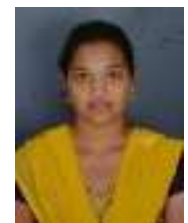

Nanduri V S S Ramya Jyothi received Diploma in Electronics and communication Engineering (DECE) from AANM and VVRSR polytechnic, Gudlavalleru, B.Tech from Laki Reddy Bali reddy college of engineering(LBRCE) and M.Tech specialization in Computers and communication engineering(C\&CE) from JNT University Kakinada. 